\title{
The Requirements of the Convention on Biological Diversity and its Effects on the Contracts of Access to Genetic Resources
}

\author{
Ebrahim Abdipour Fard ${ }^{1 *}$, Seyed Jafar Mousavi ${ }^{1}$ \\ ${ }^{1}$ Faculty of Law, Qom University, Qom, Iran \\ Corresponding Author: Ebrahim Abdipour Fard, Associate Professor, Qom University, Faculty of Law, Amin Blvd. Qom, Iran. Email: \\ Drabdipour@Yahoo.com
}

Received May 2, 2018; Accepted October 26, 2018; Online Published December 20, 2018

\begin{abstract}
The implementation of the convention on biological diversity (CBD) on contracts of access to genetic resources and sharing benefits of them has led those types of contracts to be away from the traditional contracts found in the law of contracts, and as a result, have found special formats to satisfy the requirements of the CBD. After studying the conditions and specific elements within the contracts of access to the genetic resources (ABS), we will find in this paper that these contracts are not consistent with any known conventional formats and are subject to the requirements of the CBD. Due to the implementation of requirements arising from the CBD as a binding international document, the principle of freedom in these contracts is faced with a number of limitations. The failure to comply with the requirements of the CBD by each party of these contracts, especially when one considers their obligations to provide grounds for the implementation of the aims of the CBD, leads to the modification or even revoking of $\mathrm{ABS}$ contracts.

Keywords: Genetic Resources, Biological Diversity, Biotechnology, Food Production

Citation: Abdipour Fard E, Mousavi SF. The requirements of the convention on biological diversity and its effects on the contracts of access to genetic resources. J Appl Biotechnol Rep. 2018;5(4):131-138. doi:10.29252/JABR.05.04.01.
\end{abstract}

\section{Introduction}

The need to provide food, health, and the development of public welfare has caused an increasing attention to carrying out research in the field of biotechnology with the aim of providing better products and achieving new methods both in the production foods and the production of medication. With the aim of facilitating access to genetic resources in the countries that have these resources, and fair and equitable benefit-sharing arising from the use of these genetic resources and biodiversity, an assembly consisting representatives of some countries made an attempt to ratify the convention on biological diversity (CBD) in $1972 .{ }^{1}$

In order to contribute to the countries, individuals, and local communities in the conservation of their genetic resources and their traditional knowledge, in its Article 1, the CBD recognized the sovereignty right of states over their genetic resources. This is while in other international instruments such as International Treaty on Plant Genetic Resources for Food and Agriculture (Article 1), genetic information of individuals is considered as the common heritage of mankind. In accordance with paragraph 4 of Article 15 of the CBD, any access to genetic resources of the member countries shall be subject to mutually agreed terms (MAT), with prior informed consent (PIC) of the contracting parties that have such resources. Accordingly, contracts with the subject of access to genetic resources and fair and equitable benefit-sharing from genetic resources came into effect.

Due to their novelty and the lack of proper strategy by the member countries from one side, and the requirements arising from the CBD from the other side, these access contracts have their own special nature and requirements. Thus, freedom of will in the signing the contracts in this category of contracts is faced with numerous restrictions. Therefore, in order to evaluate the effects of the requirements of the CBD on these contracts, we will explain the special conditions of the contracts of access to genetic resources (ABS), and study the legal nature of these types of contracts in section 1 . Then in the section 2, we will examine the requirements of the CBD and its effects on these contracts. It should be noted that by the requirements of the $\mathrm{CBD}$, we mean those requirements that legally affect contracts.

The Special Elements and the Legal Nature of the Contracts of Access to Genetic Resources

Special Elements of the Access Contracts

The full title of access contracts is "Access to Genetic Resources, and Sharing the Benefits, arising of their Use". Some other texts also mention it as Material Transfer Agreement (MTA).

Copyright (C) 2018 The Author(s). This is an open-access article distributed under the terms of the Creative Commons Attribution License (http:// creativecommons.org/licenses/by/4.0), which permits unrestricted use, distribution, and reproduction in any medium, provided the original work is properly cited. 
It should be noted that if contracts and agreements of transfer of resources are concluded by member countries or their citizens, they are subject to the terms of contracts for access to genetic resources and sharing the benefits arising of them.

\section{Delivery of Resources to the Users}

The original aim of the ABS contracts is to provide resources for the exploitation and carrying out research and development in order to obtain information, technology and the production of new materials. In accordance to the paragraph 4 of Article 15 of the CBD, any access to genetic resources shall be subject to PIC of the contracting party that provides these types of resources including country of origin or source country (CBD, article 15-2). So the CBD has used the term "access" because the ownership of the resources is not legally transferred. On this basis, in ABS contracts, providing physical samples of the required resources in order to carry out research operations on those resources is always the inherent and main provision of these contracts. For example, see paragraphs 17(1), 18(g), 29, 30 of Biodiscovery Act of Queensland. ${ }^{2}$

\section{Carrying out Research on the Genetic Structure of the} Species

One of the most important distinctive features of the contracts on the transfer of biological genetic resources and the contracts on the access to genetic resources concerns the condition of genetic engineering operations on the genetic structure of the species discussed by the recipients of the species. While in the contract of (physical) transfer of species, the aim of recipient is not to perform genetic modification operations on those species but is only to use the carcasses of resources. Therefore, if the intention of the parties of such contracts is not to utilize the properties and genetic information of the species under contract, these contracts, are not considered as the access contracts, and as a result, will not be subject to the provisions of the CBD.

In some contracts the provider may provide access to the resources instated of delivery of resources.

\section{Maintaining the Ownership for the Provider of Genetic Resources of the Species}

Maintaining the ownership of the provider on the genetic resources and the genetic information of biological species and, even its physical body is one of the basic conditions of ABS contracts. Such that, this feature will distinguish these types of contracts from other contracts such as contracts of sale. Therefore, under this condition, the utilizer is required to transfer the carcass of the species to the provider at the end of the contract (part5, Section 33 of the Queensland's Bio Discovery Act).

\section{The Legal Nature}

Due to the presence of some governmental and international regulations and requirements, $\mathrm{ABS}$ contracts have dual nature. Due to some national considerations and international considerations regarding the provisions of the $\mathrm{CBD}$, the rule of law in these governmental regulations, as seen for example in the Article 33 of the Queensland's Biodiscovery, act have partly made these types of contracts similar to governmental contracts. On the other hand, the profit-making and commercial nature of these contracts makes these contracts appear like private and commercial contracts.

Accordingly, one can consider ABS contracts as certain contracts with their own characteristics, such that the provider of genetic resources, while keeping his or her ownership on these resources, makes these resources available to the utilizer. On his part, the utilizer of these resources uses the resources at his disposal to carry out research, to do certain modifications, like genetic modification of the structure of these genetic species within the limits of the access license issued by the country of origin of these species. On the other side, owner of the resources will have his own share in the benefits and in the results coming from the objectives of the CBD. So in order to understand the legal nature of these contracts, one must study them with its own character and the requirements arising from international conventions on the biodiversity of species.

The Effects of the Requirements of the CBD on Access and Benefit Sharing Contracts

In accordance with paragraph 4 of Article 15 of the CBD, access to genetic resources is possible in accordance with the MAT and in subject to the provisions of the Article 15 of the CBD. Therefore, ABS contracts should comply with the requirements of the convention. Thus, while explaining the requirements governing the access agreements, we make an attempt to explain their effects upon these contracts.

\section{The Need for Prior Informed Consent of the Source Country}

Under this requirement, which is a departure from previous approaches regarding the ownership of genetic resources, governments have sovereign right over the use of their resources according to their own environmental policies, the Charter of United Nations, and the principles of international law. Accordingly, in accordance with the paragraph 5 of Article 15 of the CBD, access to genetic resources shall be subject to PIC of the country that provides such resources.

Concerning the principle of state sovereignty (article 3 of $\mathrm{CBD}$ ), one must admit that the sovereignty of the states over their genetic resources, as seen in the phrases of the CBD, is conceptually distinct from their ownership of such resources. This is because different laws in countries follow different approaches regarding the ownership of these genetic resources. Some countries such as Iran bio discovery Act consider the ownership of genetic resources as belonging to the state and some other consider it as belonging to the private sector, while some others consider a combination of both approaches. So what is considered in Article 3 of the convention is not the ownership of genetic resources but the state sovereignty over these sources. In accordance with the Fifth Report on the United Nations Environment Program, dated August 2007, the sovereignty of states on access to genetic resources means that the state exercises its own authority to recognize the ownership and control over its genetic resources and the sharing of benefits in their sovereign territory. ${ }^{4}$ 
Accordingly, in accordance with the paragraph 3 of Article 15 of this convention, any access to genetic resources of the source country, regardless of the type of ownership of the resources, must be carried out with a written request, expressing the specifics and the objectives of the access, as well as the benefits and the possible risks of the relevant operation. Besides, in accordance with the paragraph 35 of the Bonn Guidelines, it is required that the relevant documents concerning the prior consent of the access contract be obtained before the document is signed. ${ }^{5}$

Although the rules against unauthorized access to genetic resources, (i.e. access without prior signing of the contract) are more transparent, and although in different countries such as Denmark, Australia, and Iran have been criminalized, it appears to be ambiguous regarding the failure to obtain an access permit. On the other hand, regarding whether the prior consent of the legal authorities must be obtained, one must seek the basis and the objectives of access permits of the relevant resources. Because as it is mentioned earlier, the main purpose of the request permissions is to recognize the possible risks of utilizing the resources that might be made by the utilizer.

Concerning the article 3 of the CBD and the paragraph 3 of its article 15, one can conclude that, due to the environmental and humanitarian consequences of genetic modification of biological species, it is necessary to obtain the prior consent of governments when the resources belong to indigenous communities or private non-governmental individuals. Therefore, in case the prior consent of the relevant government is not obtained or is breached by the utilizer the contract is unenforced due to failure to obtain such a consent and if it fails to be enforced by a governmental authority, the contract is void. This is because a condition to obtain approval by the legal authorities, due to its relevance to public order, is among mandatory laws and in the event of failure to comply with this condition, the contract will be void. For example, in accordance with paragraph 12 of article of Ethiopia Access to Genetic Resources and Community Knowledge, and Community Rights Proclamation No. $482 / 2006^{3}$ issuing license to access biological resources and traditional knowledge is only at the disposal of Ethiopian Biodiversity Institute, (state-owned), though the ownership of genetic resources in accordance with paragraph 1/5 percent belongs to the state and also Ethiopian peoples. As previously mentioned, in accordance with the paragraph 5 of Article 15 of the CBD, a condition to obtain PIC is an introduction to basic elements of a valid contract. Therefore, in case the parties sign a contract without obtaining a PIC, the contract is void based on paragraph 3 of the CBD, in case the future approval is not granted through issuing access permission to the resources embodied in the contract. ${ }^{4}$

However, in case that previous permission has been granted but terms and the provisions of the agreement are incompatible with the permissions issued, this can cause ignorance (ambiguity) in the descriptions and in the subject matter of the contract. Because an access permission determines the scope of operation and the type of resources of the subject of a contract, and in legal terms, it describes the subject matter of ABS contracts. ${ }^{3}$

Hence, in accordance with paragraph 35 of the Bonn Guidelines, the PIC must be attached to the terms agreed. On the other hand, the access request must include some necessary provisions under its paragraph 36. Among these conditions are the type and number of species, the type of use, and the purpose of the collection of those species. In case the contract is concluded, each party, in addition to obtaining access permission or PIC, must determine its contract conditions based on the requirements and provisions of access permissions. Now the question is if the parties violate the terms of access permission or if they do not obtain PIC, what will the effect be on the contract?

One might consider that depending on the type of the breach, this might result in rescission, revoking or terminating the contract for the breach of the conditions of contract or misrepresentation. ${ }^{4}$ As the provisions of the permission are inserted in another provisions of the agreement and as a result, due to the violation of the contract, the provider is able to revoke the contract or terminate it. ${ }^{5}$

In addition to what is mentioned above, the logic behind obtaining PIC is the exercise of the sovereignty of the state on the sources and species under its authority; and each party is obliged to comply with its terms and conditions. Accordingly, in such cases, it is the legal authorities, who will decide on the maintenance, revocation or modification of the contract in accordance with the provisions of the Bonn Guidelines and Article 3 of the convention. ${ }^{1,6}$

Therefore, the obligation to obtain PIC prior to concluding the contract will have its effect on the legal status of the contract both in terms determining the scope of the obligation of each party and in terms of the principle of state sovereignty over the provisions of ABS contracts and its amount of benefit-sharing. The legal remedy of violating the terms of the access permission is applicable not only to the terms of the agreement, but also to the failure to comply with the requirements arising from the principle of state sovereignty over the resources and species under its territory in accordance with Article 3 of the CBD.

Therefore, the beneficiaries in the breach of PIC conditions are not only the parties to the contract, but also the relevant legal authorities. Therefore, the principle of state sovereignty over its resources and the principle of the need to obtain PIC of the source country are two interrelated concepts. ${ }^{7}$

Additionally, as mentioned above, the necessity to obtain access permission prior to concluding the contract has its own legal effects in the framework of the law of contracts. This is because obtaining the PIC condition is an introduction to the acceptability of the essential elements of a valid contract under paragraph 5 of Article 15 of the CBD. In general, among the purposes of obtaining an access permission to resources are the full preparation of the providers of genetic resources for an accurate evaluation of the nature of the resources, the potential and actual value, and the potential use of these resources before the owner of the resource declares consent to collect the species and, finally, to know the potential risks of the operations and the desired results of the applicant (Paragraph B of Article 5 of the Universal Declaration on the 
Human Genome and Human Rights). ${ }^{8}$

This is while in the field of contract law, the necessity of such a requirement is enacted on the basis of truth conditions of a contract with the aim of supporting both parties (especially the owner of the resources as the weaker party) against fraud or mistakes in the subject matter of the contract, failure to understand the concepts of the contract, and deliberate or unintentional errors in disclosing the information within the contract.

Apparently, any provider whose consent on the basis of the full evaluation of the contract has not been obtained is in fact uninformed of the subject matter of the contract; therefore, any such contract is void or revocable. For example, in a case where the subject of the contract is an extensive collection of forest species, merely mentioning that the subject of the contract is the genetic information within the cells of the forest species, makes this type of contract, due to the existence of ambiguity in the subject matter of the contract and the failure to match the intentions, void from the point of view of the CBD and the law of contracts. ${ }^{4}$

Principle of Access to Genetic Resources Based on the Mutually Agreed Terms by the Parties

The most important effect of the CBD on access contracts is the prediction of special conditions in these types of contracts. Because, as already mentioned, by explicitly mentioning the terms and conditions, the members of the convention have recognized the sovereignty of the countries on the resources above. In accordance with the paragraph 4 of Article 15, access to genetic resources, if granted, shall be based on MAT of the provision of this article. This condition is required for the Queensland's Bio Discovery Act.

The agreed terms between the two parties are the most important means by which the obligations of the utilizer towards the provider of the species are specified. In the context of the provisions of the convention and its appendices, the term "agreed terms" is a combination of both legal and contract terms, which will create transparency in the responsibilities of the utilizer. ${ }^{4}$ Accordingly, any failure to comply with the principle of access in accordance with agreed terms can take place in 2 ways:

The first is that access is made without any agreement or signing of the contract. In this case, the laws in different countries, such as the Queensland's Bio Discovery Act, India, Norway, Denmark, as well as the Iranian law, have considered this matter as a criminal offense, subject to criminal penalties. Such punishments are considered as guarantees for cases of failure to sign contracts regarding the biological species.

In the second way, despite the relevant contract is concluded, the utilizer imposes his or her own conditions upon the provider in such a way that the aims of the CBD are overseen. This is while the aim of the CBD to explicitly mention the foregoing conditions should not be under the influence of stronger countries, so that the weaker countries, which suffer from weaker situations, can insert their own conditions in to the contract. ${ }^{9}$

In this way, the provider may, in accordance with the requirements of the $\mathrm{CBD}$, appeal to the court to revoke or modify the contract, because the access has not been made on the basis of the full consent of the provider. Therefore, with regard to the emphasis made by paragraph 4 of the Article 15 of the CBD concerning the need for a freedom of the parties of the agreement on access to genetic resources, in the event of the imposing any conditions against the provisions within the CBD, the contract may be subject to modification or revoking, depending on the case.

However, the necessity of concluding of the contract in accordance with the terms of the agreement between the two parties does not mean the full freedom of the parties or the full implementation of the principle of free will. By accepting the above principle, however, the contract itself will be subject to the terms and conditions of the convention, because under paragraph 4 of the Article 15, the agreement by the two parties on the access should be subject to the conditions of that article and not to impose restrictions that run counter to the objectives of this convention (Article 15, 2).

\section{The Principle of Facilitating Access to Genetic Resources} Contrary to the issue of biological resources, which are recognized by national laws in different countries, the ownership of genetic resources still suffers from ambiguities. In more explicit terms, biological resources include the physical components of biological species, while genetic resources contain information about the genetic structure of biological resources. So the physical components and carcass of biological resources may be depending on the case, be owned by individuals or by the state, while the genetic information of these resources may belong to some other individuals. ${ }^{10}$

The issue of access to genetic resources of different countries is considered as a tool for achieving the three objectives of the CBD. In accordance to Article 1, the realization of the three objectives of the convention is possible through the appropriate access to genetic resources and the proper transfer of the related technologies. On the other hand, in the introduction section of the $\mathrm{CBD}$, access to the resources and the shared utilizations of them in line with the aims of the convention is considered a crucial factor. Therefore, since the paragraph 4 of the Article 15 considers access to genetic resources of different countries subject to the conditions agreed upon by the parties, in the contracts for access to biological species, which most of the governments sign with companies active in biotechnological activities, the owners of resources are obliged to observe the principle of facilitating access to resources. The Nagoya Protocol, ${ }^{11}$ which aims at fair and equitable benefit-sharing of utilizing genetic resources through appropriate access to the genetic resources and appropriate transfer of the relevant technologies, in its Article 1 considers facilitating access to genetic resources by member states as a binding rule for the owners of the resources.

Therefore, the question is in accordance to the provisions of the paragraph 4 of Article 15, which allows access only by mutual agreement by the parties, if in a contract the right of access to resources has been prevented in some way or other, 
or has been made difficult by including conditions in the contract, then what effect will this matter on access contracts for biological species?

In other words, in case there are distractive conditions on access to genetic resources, will the utilizer in these types of contracts be able to appeal to the court by resorting to the provisions embodied within the contract to bind the provider to facilitate the access to these resources in accordance with the regulations of the CBD, especially under Article 15?

In accordance with paragraph 2 of Article 15 of the $\mathrm{CBD}$, countries are bound to facilitate access to the genetic resources. Similarly, under the same paragraph, countries are prohibited to impose any restrictions that are against the objectives of the convention.

However, in case such a contract is signed between two parties, but in accordance with the provisions of the convention, access to the genetic resources is restrictive and against the principle of access to genetic resources, the issue remains ambiguous due to its failure to observe a fixed and reliable procedure. Nevertheless, one can also find an appropriate legal solution by reference to other articles of the $\mathrm{CBD}$, especially its article 15 .

In this way, the court can modify or amend the contract and consequently oblige the provider to facilitate access by the utilizer. However, it is on the court or the legal authority to determine the instances of limited or fair and equitable access to these genetic resources.

The Principle of Facilitating Access to the Interests and its Fair and Equitable Benefit-Sharing

In addition to the access to the resources, access to the benefits resulting from carrying out business on these genetic resources by the counties, is still another important objective of the convention. In accordance with paragraph 1 of Article 16, each member country, knowing that the term "technology" also includes "biotechnology" and the transfer of technology between the member countries is intended to achieve the objectives of the convention, is obliged to provide facilities for other member countries to have access to technologies relevant to the inexhaustible protection and utilization of these genetic resources. Access to the benefits of using genetic resources of the countries is the most important tool for achieving the objectives of the CBD.

Obviously, given that transfer technology, as the most important result of the use of genetic resources, should be subject to the agreement between the two parties and must be based on the contract between the two member states, the conditions and the provisions of the convention will be governed on them, especially its paragraph 2 .

It should be noted that according to the convention, by "benefit" it does not simply mean financial benefit resulting from utilization of genetic resources; it also includes access to biotechnology, educational services, and other similar benefits. Nonetheless, what has been most intended by the members is access to biotechnology. As a result, members have specifically addressed the issue of access to technology, in accordance with Article 16 of the Convention. The issue of access to genetic interests and, more precisely, biotechnology appears to be more complicated because of its relevance to The Agreement on Trade-Related Aspects of Intellectual Property Rights (TRIPS).

Now the question is if the contract is implemented, but due to the international nature of the access contract, the utilizer of the resources by referring to TRIPS and on the basis of his or her exclusive rights over the scientific and intellectual effects of implementing the contract, refuses to provide access to the provider, or refuses to grant access to the indirect benefits resulting from the implementation of the contract, what legal rights the provider of the resources will have against the avaricious claims made by the utilizer.

The answer to this question requires a proper understanding of the position of the CBD and the TRIPS on intellectual property resulting from the implementation of the operation to modify the genetic structure of biological species.

Nonetheless, regarding the relationship between the TRIPS and the $\mathrm{CBD}$, there are different ideas on their impact on access and exploitation of innovations of biological resources which goes beyond the scope of the present study. But because of the importance of this issue, we will briefly mention the main discussion in defining the laws governing the access to intellectual property from genetic resources.

From the perspective of some lawyers, the TRIPS and CBD lack inherent conflict of ideas as each includes its own objectives. These lawyers for the access to intellectual properties resulting from the application of biological resources, there is on need observe the terms and conditions of the TRIPS, since it goes against the objectives embodied within the CBD. From this point of view, TRIPS can be of help for the achieving the aims of the CBD. In the end, if there are any ambiguities, an international decision should be done in this field.

From the perspective of some others, based on article 27(3) of TRIPS, countries are free to select their patent systems in genetic innovations, so they may ignore the rights of countries on their genetic recourses in such innovation by no mention the name of the origin or provider country or other requirements such as MAT and benefit sharing instruments. This is while the CBD recognizes the authority of the governments over their biological resources as a main principle. So, from the point of view of these lawyers, TRIPS does not recognize the terms and conditions of the CBD, like the prior consent of the provider and the fair and equitable sharing of the benefits of the biological resources.

By careful studying the content of the $\mathrm{CBD}$, like paragraph 5 of Article 16, one can conclude that the approach taken by members not only fails to set preferences of TRIPS over CBD, but also according to this group of lawyers TRIPS and other international documents must be applied in such a way that they can protect the objectives of the CBD. Therefore, in case there is any uncertainty or ambiguity, the regulations of the TRIPS must be applied in such a way that they do not violate the terms and conditions of the CBD. ${ }^{12}$

On this basis, if we admit this idea that believes that the CBD in these cases has preferences over TRIPS, the utilizer of the resources has to set his or her rights such that they do not result in challenges to the $\mathrm{CBD}$, and to the rights of the 
provider of the resources. Therefore, the provider is obliged to provide facilities for the utilizer to have access to the benefits from the resources. In the same way, the benefits, especially those resulting from the relevant technology, should be shared in a fair and equitable manner (paragraph 2 of Article 16).

Another point to be mentioned about the fair and equitable sharing of benefits resulting from the genetic resources which concerns the present trend in the law of the contracts is the lack of economic balance of the parties which has its own drawbacks like the freedom of the parties to rescission the contract on grounds of lacking proper benefits from the contract. Does the fair and equitable sharing of benefits have its guarantee of its proper effect apart from those available in the traditional contracts?

The fact that what types of effects the lack of observing the provisions of the $\mathrm{CBD}$, including its fair and equitable sharing of benefits has on these types of contracts is something that has less been taken into consideration. This issue has its roots in the business-type mentality on these types of contracts on the one side, and the similar approach taken by the parties of the contract over these types of contracts and over other types of contracts, on the other. Nonetheless, the access and benefit sharing contracts that are signed in accordance to the paragraph 4 of Article 16, and paragraph 2 of Article 16, should necessarily provide the objectives of the CBD, including the fair and equitable sharing of benefits of the genetic resources. Similarly, in the introductory part of the Nagoya Protocol, as an appendix to the CBD, and in paragraph 14 (section 5) and especially paragraph $9 \mathrm{~b}$ of Article 16, the guarantee of fair and equitable distribution of benefits resulting from the utilization of genetic resources, including the transfer of technology to the country that provides the resources, has been decided as an obligation for the member states.

For example, if the utilizer in the contract refuses to transfer the technology to the provider of genetic resources and merely contributes to the provider in part of the revenue and income resulting from commercialization of the benefits, then it appears to be the case that the fair and equitable sharing provision is not observed according to the objectives of the convention. In these circumstances, the provider may, on the basis of the provisions of the convention, especially Articles 1 and 16, appeal the modification of the contract or even revoke the agreement between the two parties in order to oblige the user to transfer the technology or to properly disclose the information embodied within the contract and oblige the user to provide other benefits such as transfer of technology and the full disclosure of information derived from use of the species mentioned in the contract. ${ }^{13}$

As such, the question is, what rights does the provider have regarding the lack of fair and equitable sharing of the benefits in the contract, and what guarantee does the provider have for its implication by the utilizer?

Concerning the effects of failure to observe the condition of fair and equitable sharing of benefits, the convention and its annexed documents appear silent. However, in accordance with paragraph 4 of Article 15, access to genetic resources is subject to conditions agreed upon between the parties. On the other hand, the requirements resulting from the convention following Articles 15 and 16 must be taken into consideration in the laws of the countries. Therefore, in the case of silence of the contracting parties in this regard, a suitable legal solution can be obtained by referring to the provisions of the convention and the unconditioned rights.

Accordingly, as previously mentioned, the issue of fair and equitable sharing of benefits of the use of genetic resources is one of the three main objectives of the convention. Since a majority of countries are members in this convention, observing its provisions in all decisions, legislations and the agreements concluded by the member states is obligatory in accordance with Article 17 and paragraph 3 of Article 16. So in case the requirements of the convention in the access contracts to the genetic resources is violated, the court can modify these types of contracts due to the failure to observe the requirement to fair and equitable benefit-sharing by countries or their citizens. However, the subject of modification or even revoking them is more related to the implementation of the contract. Because if the genetic structure of the resource has been analyzed and the user has got their information, then revoking the contract is not an effective guarantee of its implementation for safeguarding the legal rights of the provider of the resources especially if the information of resources is disclosed in user country. Therefore, in this case, according to supportive approach adopted by the convention, the court should take a modification approach for the conditions of the contract. Even in such cases, some courts first issue a verdict to revoke the original contract and then oblige the parties to conclude a new contract in order to safeguard the legal rights of the provider. ${ }^{13}$

Now the question is if the court decides to modify the contract in order to share the benefits of the parties in a fair and equitable manner, on what criteria should this be carried out? Generally, the theories concerning this issue are divided into two general categories:

The first category of theories is based on the legal rights of the parties, and is based on paying off to the owners of resources and the owners of genetic resources. In this category of legal theories, attempts are made to create a kind of legal right on the genetic resources and the traditional knowledge for the owner of the resources, being states, indigenous communities, or private individuals, and find a sharing of these benefits to these resources. ${ }^{14,15}$

The second category of the theories pays attention to the interests of the owners of the resources, and maintains that this issue by itself cannot fulfill the main objective of the CBD, namely, the eradication of poverty and maintaining constant development. Therefore, because of the situations imposed on them, one has to give more shares to the poor countries. This view is based on the principles of Desert, Need, and Equity. ${ }^{16,17}$

Accordingly, the important issue in the explanation and the interpretation of the concept of justice in the access contracts to genetic resources concerns which of the theories above can more effectively serve the purpose of the CBD. By referring to the provisions of the convention, one can conclude that, it is not the aim of the convention to merely provide grounds for paying off to the owner or the owners of resources, especially to the holders of technology, but its aims are the fair and 
equitable distribution of benefits, eradication of poverty, and meeting the basic needs of less developed countries, such as training, food, heath care, and medication. Therefore, if one considers the two approaches above, and integrates the two theories into one single practice, especially by taking an approach to the supplying of the needs for these communities, then we can achieve the criteria to apply the notion of fair and equitable distribution of benefits in the access contracts to the genetic resources.

For example, the issue of access to technical knowledge in Article 16 of the CBD is considered as one of the means of the eradication of poverty and the protection of the environment. Therefore, if the provider of the resources, due to his or her role in making technical knowledge and intellectual property resulting from the use of genetic resources, makes it difficult for the provider to access to the technical knowledge or refrains from fully disclosing the required information, then this may result in a modification of the contract in contravention of the provisions of the $\mathrm{CBD}$, particularly the first paragraph of Article 16 of the CBD, or it may result in obliging the utilizer to fully disclose the information to the provider of the resources. This is because in accordance with paragraph 1 of Article 16 , access to the required technology is among the essential elements for achieving the objectives of the convention. On the other hand, in accordance with paragraph 2 of the same Article, access to biotechnology for the developing countries should be subject to fair and equitable situations. ${ }^{18,19}$

\section{Iranian Law}

Although Iran has joined to the CBD in 1996, in respect of ABS contracts there is no effective law or legal proceeding as yet. Although enacting the law of protection and utilization of genetic resources recently, this act has delegated this task to the state organs. So there is no proceeding in ABS contracts in Iran. But under article 9 of the civil code of Iran the CBD is as a national law and Iranian court must act based on CBD. So any contracts or any type of access to genetic resources in this country must be based on requirements of CBD and related instruments such as Nagoya Protocol on Access to Genetic Resources and the Fair and Equitable Sharing of Benefits Arising from their Utilization to the CBD.

\section{Conclusions}

Contrary to traditional law of contracts, in accordance with paragraph 4 of Article 15 of the $\mathrm{CBD}, \mathrm{ABS}$ contracts are subject to the requirements of the CBD. Therefore, these types of contracts, in addition to fulfilling the needs of the parties, should also fulfill the objectives of the CBD as portrayed in Article 1.

Consequently, compared with traditional contracts, ABS contracts are considered as special types of contracts with a combination of voluntary and compulsory provision with the aim of providing access to genetic resources. In the member countries of the CBD, it is subject to the same requirements. Due to the requirements of the CBD and its appendices like the Nagoya Protocol and the Bonn Guidelines, these contracts can be considered as certain types of contracts with their own legal nature.

In order to fulfill the objectives of the CBD, observing its obligations and its requirements is considered binding on the member states, especially in the private contracts. Accordingly, any breach of the requirements of the CBD by the member states or individuals and companies in the ABS contracts results in the modification or revoking of these contracts depending on the conditions embodied in each contract.

Also, according to the principle of fair and equitable sharing of benefits as a way to achieve the goal of the eradication of poverty and sustainable use of genetic resources, the concept of justice in this category of contracts is different from the one found in the theory of commutative justice in another contracts. Based on the aim of eradication of poverty found within the convention, one can conclude that the approach of the CBD concerning the concept of justice is based on a combination of theories of the entitlements of these countries and individuals on their biological species. Additionally, the concept of justice in this convention is based on the principle of need, fairness, and equitability in order to eliminate poverty from the less developed countries and communities. On this basis, the concept of justice and the lack of economic benefits in these types of contracts have an idea beyond the concept of commutative justice as lack of financial benefits in the contracts by the parties. Therefore, one can modify or even revoke the contract on the basis of fair and equitable sharing of benefits apart from each party's right to rescission the contract due to their economic disadvantages.

Contrary to the traditional contracts the necessity for PIC of the countries has led these categories of contracts to be subject to certain non-contractual requirements, including the requirement to grant approval by their governments (PIC) or concluding MAT for access to genetic recourses. Any failure to fulfill such requirements could lead to the revoking of the contract by the governmental authorities. On the other hand, non-compliance of contract with the terms of the access permission may result in cancellation or revoking the contract due to the mistake in the subject matter of the contract or some of its terms and conditions as a guarantee of its implementation.

In Iran's legal system, there is still no specific procedure in this regard and despite the requirement of paragraph 7 of Article 15, and paragraph 3 of Article 16 of the CBD, to provide and facilitate its legal implementation, one can hardly find any effective legislative measures in this field. Nevertheless, in accordance with Article 9 of the Iranian Civil Law, in the event of the signing any contract of access to genetic resources, it is subject to the provisions of the CBD, and therefore, in the process of setting any such document or interpreting it, one has to take into account the principles and requirements of the $\mathrm{CBD}$.

Authors' Contributions

All authors equally contributed to the current study.

Conflict of Interest Disclosures

The authors declare they have no conflicts of interest. 


\section{References}

1. Convention on biological diversity. United Nations; 1992. https:// www.cbd.int/doc/legal/cbd-en.pdf.

2. Queensland's Biodiscovery Act, 2004. https://www.legislation. qld.gov.au/view/html/inforce/current/act-2004-019.

3. Ethiopia Access to Genetic Resources and Community Knowledge, and Community Rights Proclamation No. 482/2006. https://www. wipo.int/edocs/lexdocs/laws/en/ug/ug001en.pdf.

4. UNEP/ CBD/ WG-ABS/5/5 30 August 2007, pp. 4-37. Https:// www.cbd.int/doc/meetings/abs/abswg.

5. Shakeel Bhatti, Santiago Carrizosa, Patrick McGuire, Tomme Young. Contracting for ABS: The Legal and Scientific Implications of Bioprospecting Contracts, IUCN Environmental Policy and Law Paper No. 67/4, IUCN Publications Services, 2009, Rue Mauverney 28, 1196 Gland, Switzerland. www.iucn.org/ publications.pp61-67.

6. Tully S. Bonn Guidelines on Access to Genetic Resources and Fair and Equitable Sharing of the Biodiversity act 2004, Queensland. RECIEL. 2003;12(1):84-98.

7. Morgera E, Tsioumani E. The Evaluation of Benefit Sharing Linking; Biodiversity and Community Livelihoods. Review of European Community and International Environmental Law. 19(2):150-173.

8. Universal Declaration on the Human Genome and Human Rights. http://www.unesco.org/new/en/social-and-human-sciences. Published 11 November 1997.

9. Accessing and benefit sharing the influenza viruses: the sting in the conflict Between CBD and TRIPS DR. Charles Lawson Australian Centre for Intellectual Property in Agriculture Griffith Law School, Griffith University, pp.5-10. http://ssrn.com

10. Convention on biological diversity UNEP/CBD-ABS/5/5 30 August
2007, fifth meeting Montreal. https://www.cbd.int.

11. Nagoya protocol on access to genetic resources and the fair and equitable sharing of benefits arising out of their utilization, 2011. https://www.cbd.int.

12. world trade organization, IP/C/W/368/Rev.1 ,8 February,2006, (060534) Council for Trade-Related Aspects of Intellectual Property Rights pp.3-10. https://www.wto.org/.

13. Morten Walløe Tvedt and Tomme Young. Beyond implementation of the fair and equitable sharing commitment in the CBD, ABS Series NO 2, p.39, 2007, World Conservation Union, IUCN, Gland, Switzerland in collaboration with the IUCN Environmental Law Center, Bonn, Germany. http://www.icun.org/Publication.

14. Hamilton C. Biodiversity, biopiracy and benefits: what allegations of biopiracy tell us about intellectual property. Dev World Bioeth. 2006;6(3):158-173. doi:10.1111/j.1471-8847.2006.00168.x.

15. Schroeder D. Benefit sharing: it's time for a definition. J Med Ethics. 2007;33(4):205-209.doi:10.1136/jme.2006.016790.

16. Feinberg J,, Narveson J. The nature and value of rights. J Value Inq. 1970;4(4):243-260. doi:10.1007/bf00137935.

17. Barry B. Access to genetic resources and sharing the benefits of their use, Political argument. London: Routledge and Kegan Paul; 1965.

18. De Jonge B. What is fair and equitable benefit-sharing? J Agric Environ Ethics. 2011;24(2):127-146. doi:10.1007/s10806-0109249-3.

19. African model legislation for the protection of the rights of local communities, farmers and breeders, and for the regulation of access to biological resources. https://www.wipo.int/edocs/ lexdocs/laws/en/oau/oau001en.pdf. 